\title{
Mass Spectrometry Search Tool (MASST)
}

Searching mass spectra against publicly available tandem MS databases just got easier.

\begin{abstract}
A huge body of untargeted mass spectrometry (MS) data is being generated through metabolomics and natural product research, much of which is publicly available in MS databases. However, searching for useful information from these datasets is a challenge. Existing methods allow searching for possible matches against a reference library of known molecules, but this can limit the search and frequently prevent discovery of novel compounds. Pieter Dorrestein and colleagues from the University of California, San Diego, compare the current state of MS-spectrum-based discovery to pre-BLAST (Basic Local Alignment Search Tool) sequence searches, when simple searches to unknown sequences were slow and ineffective. The development of BLAST was instrumental in making
\end{abstract}

sequence searches the norm in many areas of biological research.

In a collaborative effort, the Dorrestein lab has developed the web-based Mass Spectrometry Search Tool (MASST), which allows one to search a tandem MS (MS/MS) spectrum of small molecules against public metabolomics repositories for identical or similar MS/MS spectra. Thanks to the adoption of universal MS data formats, MASST can search against various repositories, such as GNPS/MassIVE, Metabolomics Workbench, MetaboLights or a combined non-redundant MS/MS library, and connect each MS/MS spectrum to its metadata entries. The userspecified parameters allow searches to find conservative matches or distant analogs.

MASST addresses several important biological questions and makes comparisons across research areas. These include but

\begin{abstract}
are not limited to comparison of molecular features in different clinical cohorts, identification of environmental toxins and their distributions, identification of chemicals in agricultural research, and microbial natural product research. The web tool provides features for easy data and parameter management and sharing, as well as the option to reanalyze results when new MS/MS libraries become available. The authors envision MASST serving an instrumental role for MS searches similar to that of BLAST.
\end{abstract}

\section{Arunima Singh}

Published online: 4 February 2020

https://doi.org/10.1038/s41592-020-0743-X

Research paper

Wang, M. et al. Mass spectrometry searches using MASST. Nat. Biotechnol. 38, 23-26 (2020).

\section{SCIENTIFIC REPORTS}

natureresearch
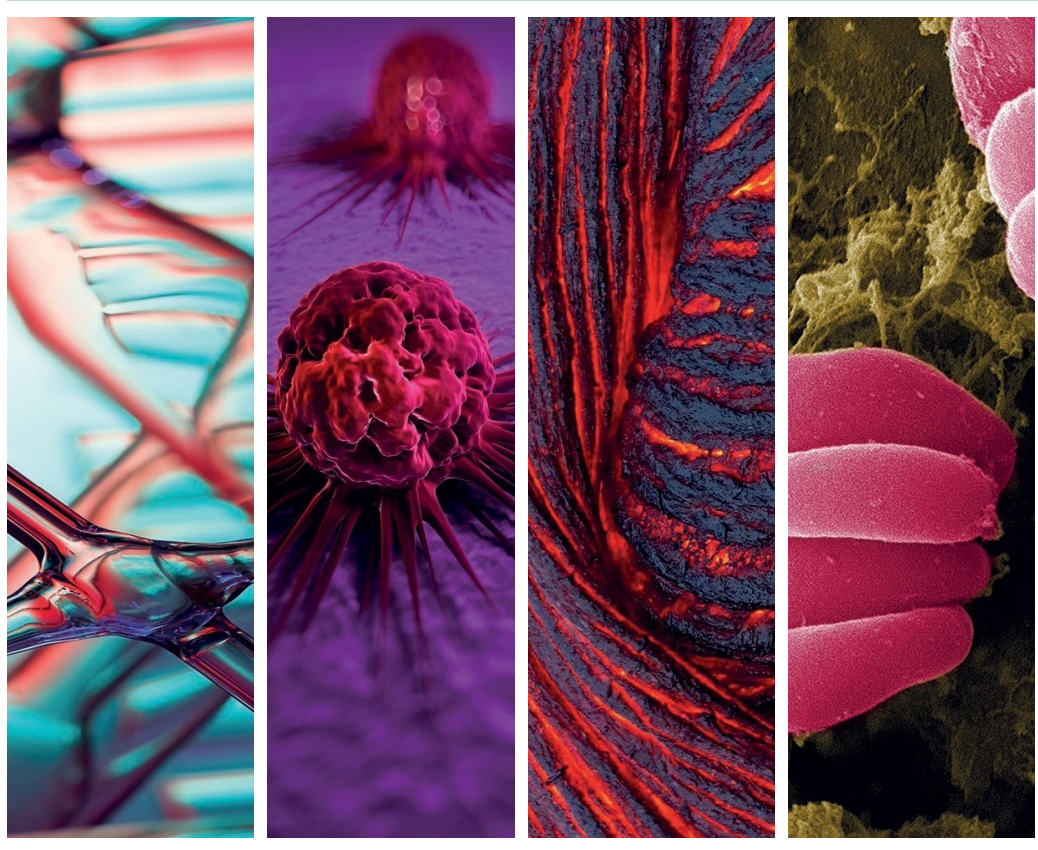

Scientific Reports is an open access journal publishing original research from across all areas of the natural and clinical sciences.

\section{As a leading multi-disciplinary open access journal with over 1.5 million readers a month, Scientific Reports is the perfect place to publish your research.}

- Expert Editorial Board to manage your paper

- Follows Nature Research's high peer review standards

- Indexed in Web of Science, PubMed and other major repositories

- Research accessed from over 180 countries worldwide 